\title{
Treatment Related Cognitive Impairment in Pediatric Oncology Patients: A Brazilian Experience
}

Izabel Hazin ${ }^{1 *}$, Danielle Garcia ${ }^{1}$, Ediana Gomes ${ }^{1}$, Bruna Balaban Garcia ${ }^{1}$, Débora Sunaly ${ }^{1}$, Amanda Guerra ${ }^{1}$, Carolina Vilar ${ }^{1}$, Francisco Pedrosa ${ }^{2}$ and Arli Pedrosa $^{2}$

${ }^{1}$ Universidade Federal do Rio Grande do Norte (UFRN), Brazil

${ }^{2}$ Integral Institute of Medicine, Center of Hematology and Pediatric Oncology - CHPO, Brazil

*Corresponding author: Izabel Hazin, Universidade Federal do Rio Grande do Norte, Brazil, Tel: 55 8488491970; E-mail: izabel.hazin@gmail.com

Received date: Apr 03, 2014, Accepted date: May 20, 2014, Published date: May 27, 2014

Copyright: ( 2014 Hazin I, et al. This is an open-access article distributed under the terms of the Creative Commons Attribution License, which permits unrestricted use, distribution, and reproduction in any medium, provided the original author and source are credited.

\begin{abstract}
Background: Acute Lymphoblastic leukemia (ALL) and Posterior Fossa Tumors (PFT) are the most common types of cancer in Brazilian children. Parallel to the sophistication of treatment and increased survival rates, the concern about the neurotoxicity of anti-neoplastic treatment and its impact on neurodevelopment has been growing. The objective of this study was to compare the cognitive impact of treatment modality on intellective capacity of children survivors of PFT and ALL.
\end{abstract}

Method: 22 Children and adolescents aged from 6 to 14 years old have participated in this study, being 06 children diagnosed with Astrocytoma, 06 diagnosed with medulloblastoma and 10 diagnosed with ALL. Children with astrocytoma underwent surgery for tumor resection; children with medulloblastoma were submitted to surgical resection, systemic chemotherapy and cranial and neuraxis radiation therapy (54Gy) and; children with ALL underwent systemic and intrathecal chemotherapy. Intellectual functioning was evaluated through WISC-III, and performance of subgroups was compared using One Way ANOVA followed by post hoc LSD.

Results: Children with astrocytoma obtained average performance in all assessed domains, while children with medulloblastoma and ALL obtained downgraded scores. In all indices children with ALL obtained better results than children with medulloblastoma, except on verbal scores (VIQ and $\mathrm{VCl}$ ) and Freedom from Distractibility Index (FDI). Statistical analysis revealed significant contrasts in performance of groups, mainly between children with medulloblastoma and other groups in non-verbal scores, as Processing Speed Index (PSI).

Conclusion: Data suggest that the combination of surgery, systemic chemotherapy and radiotherapy may intensify the incidence of cognitive effects. Moreover, reinforce the hypothesis that cranial radiation therapy underlies severe cognitive losses, through damage to cortical and subcortical white matter, so that motor and mental processing speed is affected. The combination of intrathecal and systemic chemotherapy in turn promotes significant impacts on executive functioning, in particular on working memory.

Keywords: Radiotherapy; Posterior fossa tumors; Leukemia; Intellectual function

\section{Introduction}

Brazilian official data estimate that annually approximately 11,840 cases of neoplasias are diagnosed in children and adolescents. The north-eastern region of Brazil, reference of this study, has the second highest number of new cases per year, about 2790. In Brazil cancer is the first leading cause of death among children and adolescents aged from 0 to 19 years. Among all annual cases of cancer, approximately $35 \%$ are leukaemia, from which $75 \%$ are acute lymphoblastic leukaemia, and $15 \%$ are Central Nervous System tumours (CNS), of which $60 \%$ will occur in posterior fossa region, where astrocytomas and medulloblastomas are more often diagnosed [1].

Developing countries still face important barriers to achieve the cure of cancer, among which stand out delayed diagnosis, high rates of premature mortality and defaulting from treatment. In Brazil, this reality is even more devastating in distant from large urban areas, notably north and northeast regions, which are at a disadvantage in regard to access to health and education services. Socioeconomic discrepancies affect educational level and health of the population and have a negative impact on diagnosis and treatment of childhood cancer - often late- and, consequently, on the possibility of rehabilitation in case of survival $[2,3]$.

Despite the persistence of these difficulties in peripheral regions of Brazil, increasing survival rates of childhood cancer can also be found, although in a more discreet way if compared to large urban centres. Therapeutic efficacy obtained by combining different treatment modalities, however, can occur at the expense of adverse neurocognitive sequelae - arising both from the effects of cancer itself and from the aggressiveness of therapeutic approaches [4-6].

In children, CNS tumours are more common in infratentorial or posterior fossa region. Among the most common types of tumours are medulloblastomas, malignant primitive neuroectodermal tumour, and low-grade gliomas, among which stand out the pilocytic astrocytomas, benign neuroepithelial-originated tumours $[7,8]$.

Astrocytomas are usually associated with benign clinical course, due to the mitotic slowness of their cells and its discrete and well- 
circumscribed histological nature. These factors usually enable complete tumour resection and do not require additional antineoplastic treatments $[9,10]$. Medulloblastoma, in turn, is a highly malignant tumour with worse prognosis, whose histology nature is characterized by high cellularity, infiltration and mitotic potential, and strong propensity to spread through cerebrospinal fluid (CSF) and along neuroaxis. These factors lead to the need for more aggressive therapeutic interventions, beyond surgical resection: chemotherapy and cranial and neuraxis radiotherapy with posterior fossa boost [11].

Leukaemia's are clonal diseases originated from neoplastic transformation of bone marrow's hematopoietic progenitor cells. They acquire genetic changes, which give them a proliferative, and survival advantage or impairment of differentiation [12]. Acute Lymphocytic Leukemia (ALL) is characterized by rapid growth of lymphocytes, which are immature bone marrow specific cells. Although it affects all age groups, ALL has its highest incidence in children aged from 2 to 5 years old with a peak at around 3 to 4 years. Standard treatment for children diagnosed with ALL has been systemic chemotherapy combined with intrathecal chemotherapy, held in a period between 30 to 36 months. Despite controversies in the literature about the cognitive impacts caused by chemotherapy when administered alone, there are outlined consonant knowledge about it, especially about visual processing difficulties, visual-motor functioning, attention and executive functioning. Such impacts have been considered similar to those found in patients treated with radiotherapy, differing only in intensity and severity $[13,14]$. Specific cognitive functions changes such as attention, memory, processing speed, verbal comprehension, visual spatial abilities and visual motor operation are also related to late effects caused by intrathecal chemotherapy [15].

Although cognitive deficits are associated with the use of intrathecal chemotherapy $[16,17]$, most expressive deficits appear to result from the use of cranial radiotherapy [18]. Studies show that radiation exposure results in generalized white and gray matter loss in the brain. However, its deleterious effects focus particularly in the hippocampus region because this is a primary site of neurogenesis [19]. Accordingly, reduction of white matter volume due to the impact on myelination, vascularity and suppression of proliferation of cells, appears to be at the core of cognitive changes [20-24].

Demyelination appears within 5 months, with vascular structural changes and necrosis occurring about 9 months later. Five years after radiation, significant structural damage to the brain appeared and continued to progress. Hippocampal volume, equally affected, has continuing bilateral white matter decrease until 3 years after the radiation end [19].

Cognitive deficits found in children treated with cranial radiation are progressive and, in general, involve intellective functioning, academic performance, memory, attention and processing speed [25-27]. Such impacts culminate with the compromise of new skills and information acquisition, resulting in significant and progressive reductions in IQ scores [19].

Cognitive changes resulting from the action of intrathecal chemotherapy and cranial radiation will depend on the complex interaction of several factors, among which stand out nature, location and extent of the lesion, sociocultural insertion of the child, the quality and quantity of environmental stimulation, the age of the child on diagnosis and, consequently, maturational context of CNS. In coactuation, these variables will have an important role in the process of brain reorganization, determining the course of its development after a brain injury [18-28].

More specifically, lesional effects caused by cranial radiotherapy in pediatric population reveal important peculiarities due to the inherent complexity of a developmental condition as well as to the interposition of a pathological condition on this process, which will set fundamental deviations from the normal process of maturation and development through the incorporation of reorganization mechanisms [29].

It is noteworthy that in these pathological conditions, post-injury reorganization mechanisms themselves - through neural plasticity are compromised in children exposed to cranial radiation therapy, as white matter, which is one of the major components in this process, is impaired. Moreover, failures in the acquisition of primary cognitive skills during certain critical periods in early ages can result in important losses in future acquisition of cognitive abilities that have them as a substrate. Thus, injury caused by radiation challenges the notion that childhood is definitely marked by higher neural plasticity and recovery potential when compared to older ages $[29,30]$.

Considering the above, the objective of this study was to compare the impact of anti-neoplastic treatment modality (isolated surgery; intrathecal and systemic chemotherapy; and radiotherapy associated to systemic chemotherapy) on intellective functioning of childhood survivors of PFT and ALL.

\section{Materials and Methods}

\section{Participants}

Participants of this study were pediatric patients with PFT accompanied by CEHOPE/IMIP in Recife/PE/Brazil and ALL survivors treated at Liga Norte Riograndense Contra o Cancer and Varela Santiago Children's Hospital, both located in Natal/RN/Brazil. These children came from two States in the Northeast region of Brazil, especially from rural areas, far from the capitals.

All children engaged in previously referred oncology services by the time data were collected were evaluated, which represents a group of 31 children, formed by 21 with PFT and 10 with ALL. This group was reduced to an effective of 22 children, since were considered eligible only six to sixteen years-old participants, with primary diagnosis of ALL or PFT, being clinically assisted and without records of neither neuropsychiatric nor sensorial alterations. These 22 children were allowed to participate in this research by their parents or legally responsible tutors, through signature in written formal term of free and informed agreement. It must be noted that PFT-Astrocitoma group was only formed by children submitted to resection surgery, while PFT-Meduloblastoma group was formed by children previously submitted to surgery, chemotherapy and radiotherapy; ALL group, on the other hand, was formed by children having been previously submitted to intrathecal chemotherapy only, as a way of protecting SNC. Participants with clinical records of recurrence of leukaemia in $\mathrm{SNC}$ and/or presence of other clinical conditions that could interfere in cognitive domains under scrutiny, as Down Syndrome or epilepsy were excluded from the sample under study, together with participants showing secondary neoplasias. All this information was collected from clinical records, parent's information and records from other professional caretakers equally enrolled in the assistance of these children during medical treatment. 
Citation: Hazin I, Garcia D, Gomes E, Garcia BB, Sunaly D, et al. (2014) Treatment Related Cognitive Impairment in Pediatric Oncology Patients: A Brazilian Experience. J Nucl Med Radiat Ther 5: 174. doi:10.4172/2155-9619.1000174

Page 3 of 9

The low number of participants in the sample of this study (22 children) must be considered and justified in the context of the low incidence of the clinical condition under study in absolute numbers, combined with elevated decease rate among them, low standard of quality in local public health assistance services and places of residence far from these urban services, in the rural zone of the state. These last difficulties were particularly important in the case of survivor children out of treatment by the time data were collected.

Difficulties above mentioned in obtaining an effective sample did not allow rigorous statistical pairing of variables (like age and sex by the time data were recollected) in observed groups. On the other hand, clinical conditions under study have different peaks of incidence in terms of age.

An inferior age-limit of six years was considered in sample building of the present research, taking into account previous published data showing that children earlier diagnosed (less than five to seven years of age) as ALL or PFT showed less favorable prognostic when compared to children who were affected and diagnosed later. It must be stressed here that almost all participants were diagnosed in their first decade of life (only one participant child diagnosed as a PFT-meduloblastoma case is out of this rule). A statistical analysis performed to verify possible significant effect over dependent variables leading to differences among studied groups due to sex did not allow to abandon null hypothesis of absence of differences (Chi $2=0.028$, d.f. $=2$, $\mathrm{p}=0,986)$. It is possible to equally assume the absence of significant effect of the variable age (One-Way Anova, F $(2,19)=1,44, \mathrm{P}=0,261$ ) and age of diagnosis (One-Way Anova, $\mathrm{F}(2,19)=3,49, \mathrm{P}=0,51$ ). composed of 13 subtests that when grouped in a specific way, provide three ranges of IQ: Full Scale IQ (FSIQ), Verbal IQ (VIQ), Performance IQ (PIQ) and four Index Factors: Verbal Comprehension (VCI), Perceptual Organization (POI), Freedom from Distractibility (FDI) and Processing Speed (PSI).

\section{Data analysis}

This study adopted a cross-sectional format, aiming to identify similarities and differences on intellectual functioning among three clinical subgroups of children diagnosed with cancer, through descriptive and inferential statistical tools.

Distribution of the sample was verified by Shapiro-Wilk test and data have attended to the hypothesis of normality. Analysis of contrasts between subgroups was obtained through One Way ANOVA followed by Post Hoc LSD, aiming to identify intellective effects possibly associated with childhood cancer treatments through pairwise comparisons. In addition, it was verified this impact through MANOVA followed by Post Hoc LSD controlling the variables age at diagnosis and sex. All analyses were processed through PASW Statistics 18 software, adopting a significance level of $5 \%(\mathrm{p}<0.05)$, for rejection of null hypothesis.

\section{Results}

Table 1 presents the main clinical and sociodemographic data of participating children.

\section{Instruments}

Evaluation of intellectual functioning was carried out through Wechsler Intelligence Scale for Children-WISC-III. WISC-III is

\begin{tabular}{|c|c|c|c|c|}
\hline \multirow[b]{2}{*}{ Variables } & \multicolumn{3}{|l|}{ Groups } & \multirow[b]{2}{*}{$\begin{array}{l}\text { Comparison of Variables' } \\
\text { Distribution among Groups }\end{array}$} \\
\hline & All $(n=10)$ & Astrocytoma $(n=6)$ & Medulloblastoma $(n=6)$ & \\
\hline Gender (male/female) & $3 / 7$ & $2 / 4$ & $2 / 4$ & $p=0,986$ \\
\hline Age at diagnosis & $2-7 ;$ average $=4.1$ & $3-10 ;$ average $=6.1$ & $2-11 ;$ average $=6.8$ & $p=0,251$ \\
\hline Age in the evaluation & $6-12 ;$ average $=8.9$ & $7-11 ;$ average $=9$ & $8-14 ;$ average $=10.3$ & $p=0,51$ \\
\hline Treatment & $\begin{array}{l}\text { Systemic Chemotherapy }+ \\
\text { Intrathecal Chemotherapy } \\
\text { (GBTLI-99 Protocol) }\end{array}$ & Surgery & $\begin{array}{l}\text { Surgery, Chemotherapy } \\
\text { and Cranial and neuroaxis } \\
\text { radiotherapy } \\
\text { (Parker Protocol) }\end{array}$ & NA \\
\hline $\begin{array}{l}\text { Drugs used in } \\
\text { chemotherapy }\end{array}$ & $\begin{array}{l}\text { Methotrexate, Cytarabine } \\
\text { Dexamethasone,Prednisone,Vinc } \\
\text { ristine,L-asparagine } \\
\text { Daunorubicin,Cyclophosphamide } \\
\text { 6-Mercaptopurine } \\
\text { Cytarabine } \\
\text { Doxorubicin } \\
\text { Thioguanine }\end{array}$ & NA & $\begin{array}{l}\text { Vincristine } \\
\text { Cisplatin } \\
\text { Carmustine }\end{array}$ & NA \\
\hline Dosage of Radiotherapy & NA & NA & $\begin{array}{l}36 \text { Gy at the skull and } \\
\text { neuroaxis }+18 \text { Gy tumor } \\
\text { bed }=54 \mathrm{~Gy}\end{array}$ & NA \\
\hline
\end{tabular}

Table 1: Clinical and sociodemographic data of children participating in the study. Note: NA: not applicable. 
Citation: Hazin I, Garcia D, Gomes E, Garcia BB, Sunaly D, et al. (2014) Treatment Related Cognitive Impairment in Pediatric Oncology Patients: A Brazilian Experience. J Nucl Med Radiat Ther 5: 174. doi:10.4172/2155-9619.1000174

Page 4 of 9

Table 2 presents the average performance of the three subgroups for each score provided by WISC-III.

\begin{tabular}{|l|l|l|l|l|l|l|l|l|}
\hline & & VIQ & PIQ & FSIQ & VCI & POI & FDI & PSI \\
\hline \multirow{3}{*}{ Astrocytoma } & Mean & 111 & 104 & 108 & 109 & 103 & 110 & 101 \\
\cline { 2 - 9 } & SD & 13,49 & 11,95 & 12,44 & 13,97 & 11,65 & 12,44 & 5,82 \\
\hline \multirow{3}{*}{ Medulloblastoma } & Mean & 94 & 72 & 82 & 95 & 75 & 89 & 69 \\
\cline { 2 - 9 } & SD & 19,08 & 14,03 & 17,08 & 19,54 & 13,58 & 15,46 & 6,91 \\
\hline \multirow{2}{*}{ All } & Mean & 87 & 90 & 88 & 91 & 91 & 82 & 90 \\
\cline { 2 - 10 } & SD & 16.09 & 6.95 & 11.18 & 17.76 & 8.82 & 11.15 & 9.18 \\
\hline
\end{tabular}

Table 2: Mean performance of subjects in scores of WISC-III. Note: VIQ-Verbal IQ; PIQ- Performance IQ; FSIQ - Full Scale IQ; VCI - Verbal Comprehension Index; POI - Perceptual Organization Index; FDI - Freedom from Distractibility Index; PSI - Processing Speed Index; SD Standard Deviation.

Children with astrocytoma obtained average performance, while children with medulloblastoma and ALL obtained downgraded scores.

Table 3 presents the findings from investigation of contrasts By comparison, children with ALL obtained better results than children with medulloblastoma in all indices, except the verbal scores (VIQ and VCI) and Freedom from Distractibility Index (FDI).

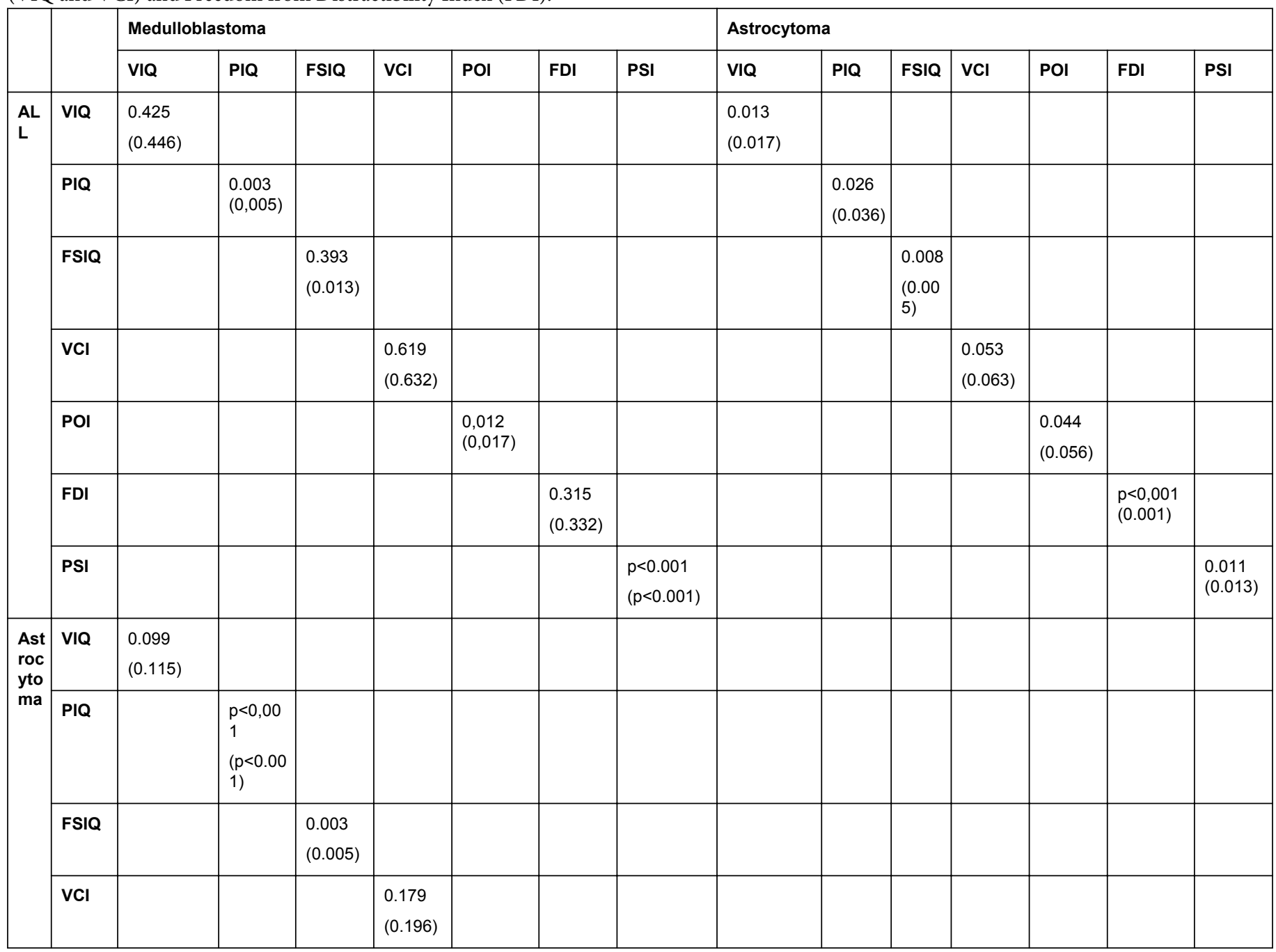




\begin{tabular}{|l|l|l|l|l|l|l|l|l|l|l|l|l|l|l|}
\hline POI & & & & & $\begin{array}{l}p<0.001 \\
(0.001)\end{array}$ & & & & & & & & & \\
\hline FDI & & & & & & $\begin{array}{l}0,008 \\
(0.011)\end{array}$ & & & & & & & & \\
\hline PSI & & & & & & & $\begin{array}{l}p<0.001 \\
(p<0.001)\end{array}$ & & & & & & & \\
\hline
\end{tabular}

Table 3: Inferential Statistical Analysis. Notes: Statistically significant differences $(\mathrm{p} \leq 0.05)$ can be verified in the values in bold. The values in parentheses relate to the results of analyses after controlling age at diagnosis and sex variables. VIQ-Verbal IQ; PIQ- Performance IQ; FSIQ Full Scale IQ; VCI - Verbal Comprehension Index; POI - Perceptual Organization Index; FDI - Freedom from Distractibility Index; PSI Processing Speed Index.

It must be emphasized that statistically significant differences in performance in WISC-III intra and among studied groups, due to independent variables age at diagnosis and sex were not observed. These statistical analysis were performed in order to verify the potential of these independent variables, frequently mentioned as relevant factors in research literature $[6,15,27]$. The absence of verified statistically significant effect here must be considered in a prudential basis, since the low number of participants mentioned above could be responsible for this conclusion. Nevertheless, supplementary statistical analysis performed with control of these independent variables show very similar results in terms of performance in WISC-III (see data in parenthesis in Table 3), with the only exception of FSIQ and POI indexes.

With regard to WISC-III verbal IQ, statistical analysis reveal significant discrepancies between astrocytoma and ALL groups, in favour of children with astrocytoma, while children with ALL had slightly below-average performance on this domain. In this area, children with medulloblastoma have not had significant contrasts with none of the groups and it is noted that this subgroup achieved average performance.

Regarding PIQ, statistical analysis revealed significant contrasts in in all pairwise comparisons. Although important contrasts were revealed on comparisons between performances of ALL and astrocytoma groups, the most evident differences were verified in the pairwise comparisons of both groups with children with medulloblastoma. This differentiation is in compliance with the following performance order: astrocytoma (classification Average); ALL (classification Average) and; medulloblastoma (classification Borderline).

With regard to FSIQ, performance order was kept: astrocytoma, (classification Average); ALL (classification Low Average) and medulloblastoma (classification Low Average). Differences between the groups ALL and astrocytoma and the latter with medulloblastoma group were significant. However, it has not been possible to verify the presence of statistically significant contrasts between groups ALL and medulloblastoma. Nevertheless, statistically significant differences were verified when age at diagnosis and sex were controlled.

The results obtained in WISC-III's Verbal Comprehension Index (VCI) revealed that all groups achieved average performance for this index and no significant differences between the three groups were found.

On Perceptual Organization Index (POI) statistically significant differences were obtained in all pairwise comparisons. These differences, however, are no more statistically significant between
Astrocitoma and ALL groups when variables age and sex are controlled (see Table 3). It should be noted that although relevant difference is observed between performance of astrocytoma and ALL groups in this index, performance of these groups are on average classification. The most significant differences were detected on comparisons among children and adolescents diagnosed with medulloblastoma and other groups. This differentiation is in compliance with the following order of average performance: astrocytoma (classification Average); ALL (classification Average) and; medulloblastoma (classification Borderline).

In Freedom from Distractibility Index (FDI) analysis revealed statistically significant contrasts between astrocytoma and ALL groups, and between astrocytoma and medulloblastoma groups. It's possible to see that the performance obtained by different groups has the following configuration: astrocytoma (classification Average) medulloblastoma (classification Low Average) and ALL (classification Borderline). A more accurate analysis of these data shows that the low score achieved by children with ALL is directly associated to the low performance in arithmetic subtest which along with digits subtest compose this index. It is noteworthy that latter subtests investigate cognitive flexibility abilities, auditory-verbal attention and working memory, which are related to executive functioning domain.

In Processing Speed Index (PSI) the results were similar to data found in PIQ and POI. Analysis revealed significant differences in all pairwise comparisons. It should be noted that astrocytoma and ALL groups achieved expected performance for this index, in spite of the verified contrasts. Main differences, however, were verified between results obtained by children and adolescents diagnosed with medulloblastoma and other groups. This differentiation is in compliance with the following order of performance: astrocytoma (classification Average); ALL (classification Average) and; medulloblastoma (classification Extremely Low).

\section{Discussion}

Wechsler Scales-WISC-III results indicate important differences on intellectual functioning of clinical subgroups studied. The best overall performance was obtained by children diagnosed with astrocytoma, followed by children with ALL and, finally, the subgroups of children with medulloblastoma.

Significant differences can be observed between performance of children with ALL and pilocytic astrocytoma in favour of the latter. Generally, children with benign tumors, as in the case of astrocytomas are treated exclusively by surgery. This therapeutic approach involves cognitive losses induced by neoplastic expansion and by mechanical 
trauma to the posterior fossa structures. However, according to medical literature, these effects are acute and susceptible by attenuation via neural plasticity, with great potential for post-injury reorganization [31].

Children with a diagnosis of ALL, however, receive intrathecal administration of chemotherapy drugs, whose associated short and long term cognitive sequel is still controversial. Neuropsychological effects more commonly found in children treated just with chemotherapy involve visual processing, visual-motor functioning, attention and executive functioning, which contribute to a decrease of performance on intelligence tests. Such data helps in the understanding of the absence of significant contrasts between ALL and Medulloblastoma groups in the WISC III full scale, but with qualitatively superior performance of subjects with ALL (treated with a combination of systemic and intrathecal chemotherapy) in relation to subjects diagnosed with medulloblastoma (treated with surgery, chemotherapy and radiotherapy).

Children diagnosed with medulloblastoma appear in this study as those with the poorer results. This data suggests that radiation therapy used for the approach of this type of tumor appear to be the most damaging to neurocognitive functioning. Studies have shown that among therapeutic modalities used in the treatment of malignant brain tumors radiation therapy is more often related to the incidence and severity of neurocognitive adverse effects [14].

Despite efforts to reduce radiation treatment toxicity, a large percentage of survival children experience academic difficulties and learning deficits. Cognitive effects found in children undergoing cranial irradiation involve intellectual functioning, academic performance (especially mathematics), memory, attention and processing speed [27].

On verbal intelligence domain, the results point to important differences in performance between children with astrocytoma and ALL, with the first group showing better performance, and no contrasts found among children with medulloblastoma and other groups. This domain involves dimensions of abstract symbols manipulation, quality of formal education and environmental stimulation, comprehension, concepts formation, memory and verbal fluency [32].

The decrease observed in children with ALL in this field is explained by significantly below-average performance identified on arithmetic subtest. This subtest investigates mathematical knowledge, but crucially depends on working memory for its execution, once children hear verbally a problem and should solve it mentally, without the support of pencil and paper. Current studies have suggested that executive functions, in particular working memory, cognitive flexibility and initiation are the weakest cognitive domains for clinical groups of children and adults survivors of ALL, both low and average risk, therefore, not exposed to cranial radiotherapy $[33,34]$.

Manipulative or non-verbal domain of intelligence has been referred as more susceptible to interference of brain injuries. This domain is used as a measure of perceptual organization, planning capacity, non-verbal knowledge and ability to think and manipulate visual stimuli with fast speed [32]. It is noteworthy the upward trend on pattern of deficits among children with astrocytoma, ALL and medulloblastoma, in this order, which indicates that the greatest losses in this domain can be observed in children who underwent adjuvant therapy on oncology treatment.
The most obvious contrasts were found on the pairwise comparisons between children with medulloblastoma and others, indicating that this group differs expressively from other subgroups, with significantly poorer results in the non-verbal domain, classified as Borderline plateau. This result is similar to the findings in literature that indicate cumulative effects of cancer treatment. Thus, although cognitive deficits associated with neurosurgery of posterior fossa tumours and systemic and intrathecal chemotherapy can be pointed, the combination of these strategies with cranial and neuroaxis radiotherapy may intensify cognitive losses $[9,14,35,36]$.

The manipulative domain of Wechsler Scales has been associated with the right hemisphere functioning. It is known that this area of the brain is richer in long fibres, which connect more distant areas in the cortex, so that it has greater axonal availability and therefore greater concentration of white matter through the myelinated fibres. Therefore, the right hemisphere, whose functioning is strongly related to non-verbal skills, can be more markedly affected by the action of radiation through the reduction of white matter, especially during childhood. This fact could be underlying to the intensity of deficits found in children with medulloblastoma in non-verbal domains $[25,37]$.

Several studies show that the radiation impact on the information processing speed lead to a slowing on the rate of new knowledge acquisition [25]. This, plus the late and progressive nature of this impact, in many cases manifests itself through decreases in IQ over time, which at first sight wrongly can lead to the belief that children lose cognitive skills previously acquired. However, previous knowledge generally is maintained, with a greater difficulty in acquiring new skills in a normal developmental rhythm. Furthermore, in children with medulloblastoma submitted to cranial radiotherapy this fact can underlie the relative preservation of the verbal domain-in the WISCIII, which is more associated with crystallized intelligence, as well as to the loss to the nonverbal domain - more associated with the fluid intelligence. This hypothesis can be enhanced by the fact that, under normal development conditions, the fluid intelligence is strongly related to the processing speed, which is impaired in children who undergo cranial radiation therapy $[25,38]$.

The four Index Factors of WISC-III reflect specific aspects of cognitive functioning. The Verbal Comprehension factor is the verbal domain in a purer and more accurate dimension [39]. Statistical analysis of clinical groups' performance on this domain did not reveal significant differences. Moreover, data show that all groups have achieved average performance in this index.

In other hand, Perceptual Organization Index is a more accurate indicator of non-verbal intelligence, enabling the assessment of visual spatial skills, non-verbal reasoning and learning, planning skills and ability to manipulate visual stimuli [39]. In this index statistical analysis revealed significant differences in all pairwise comparisons, following the same pattern found in PIQ. When age at diagnosis and sex variables were controlled, the analysis did not reveal significant differences between astrocytoma and ALL groups in this domain. The poorer performance in this domain was achieved by medulloblastoma group (classification Borderline) which showed the only lower than average performance and the highest statistical differences in relation to other groups. Impact can be related to mental and psychomotor speed and is directly related to changes in white matter density resulting from exposure to cranial radiotherapy [2]. 
Freedom from Distractibility Index (FDI) revealed statistically significant contrasts between groups. This index depends on auditory memory, verbal working memory and sequential processing, shortterm memory and concentration. In this domain children with ALL showed the poorest performance and as this factor is closely related to mathematical ability, the results revealed that arithmetic subtest underlies to the decreasing performance of ALL group in this domain. Studies that assessed post treatment cognitive performance in children diagnosed with ALL submitted only to chemotherapy corroborate this data. According to them, attention, memory and executive functioning were the most damaged cognitive domains [17,40,41]. Authors suggest that these deficits are directly related to the use of MADIT protocol (combination of intrathecal methotrexate, cytarabine and dexamethasone).

Finally, Processing Speed Index (PSI) reflects psychomotor and mental speed for solving nonverbal problems, and also the ability to plan, organize and develop strategies. These skills involve two cognitive domains, as processing skills implies both cognition and speed, and have cognitive and behavioral components. This index revealed strongly significant contrasts in all pairwise comparisons of clinical groups, with astrocytoma and ALL groups' performance on average, while children with medulloblastoma presented their worst deficits (classification Extremely Low). This result is consistent with previous findings in pediatric oncology domain, which refer to a strong relationship between the use of cranial radiation and reduction of white matter - which is involved in myelination of nerve fibers and subjacent to this factor, decreased processing speed, working memory and intellective capacity $[28,42]$.

Medicine has been seeking the improvement of childhood cancer treatment, with efforts that are adding to the concept of healing the quality of life dimension for childhood cancer survivors. In Brazil, as well as around the world, the advances and increasing sophistication of therapeutic approaches has ensured the gradual increase of survival rates. However, this survival is still followed by significant adverse effects on cognitive functioning and learning of these children.

Among the advanced therapeutic approaches against cancer, cranial radiation therapy is still one of the more efficient and effective in combating malignant CNS tumors, like medulloblastoma, which has a high incidence in childhood. Nevertheless, the replacement of radiotherapy for intrathecal chemotherapy for prevention of CNS in cases of leukemia has been widely adopted in Brazilian protocols [43].

This study was carried out in the northeastern region of Brazil, characterized by important inequalities between rural and urban areas, in particular regard to access to health and educational system. These difficulties can lead to a late diagnosis and the worsening of clinical picture, which is often followed by adverse medical conditions that make the treatment more complex. In this background, we sought to contrast the impacts on intellective capacity of survivors children of three distinct neoplasias associated with specific therapeutic modalities: Posterior Fossa Tumors- Astrocytoma (Neurosurgery) and medulloblastoma (Neurosurgery associated with systemic chemotherapy and radiotherapy) and; acute lymphoblastic leukemialow and medium risk (intrathecal chemotherapy associated with systemic chemotherapy).

The results support findings from several studies worldwide. Initially, significant impacts were not identified on the overall intellectual functioning in children with astrocytoma. Data presented in literature through systematic review are inconclusive in this regard
[44]. It was noted further that the therapeutic modality which combines intrathecal and systemic chemotherapy as CNS prevention in the treatment of ALL appears to be directly linked to specific cognitive deficits, such as attention and executive functioning, notably working memory, cognitive flexibility and initiation, without direct repercussions for the ovberall intelligence $[33,34,45]$. Finally, the combination of neurosurgery, systemic chemotherapy and cranial and neuroaxis radiation - adopted in treatment protocols for medulloblastoma, appear to intensify cognitive damage, notably by reducing the speed of information processing and directly impacting intellectual functioning of this clinical subgroup $[18,19]$.

It is suggested that the losses arising from the administration of cranial radiotherapy are enhanced by acting incisively on white matter, which is one of the main agents of neural plasticity and reorganization processes after a neurological lesion [30]. Such impact has effects in the process of myelination of white matter in specific brain areas, such as the corpus callosum [46] and hippocampus [20].

Thus, findings of this study endorse the discussion around the dynamic and multifaceted character of neurodevelopment and the dynamics of cognitive functioning during the nervous system's maturation, on the presence of toxic agents to its developing structures. These results provide important contributions to the understanding of the course undertaken by the CNS in development in the face of distinct neurological conditions, different therapeutic approaches, as well as in the context of different maturational frames and the influence of multiple clinical and sociocultural variables. In addition, alert to the need to develop therapeutic protocols that seek to minimize cognitive impact of radiation, considering protective aspects of neurogenesis when using this technique [46].

Although this study had encouraged important discussions about neurodevelopment in specific pathological conditions, some important limitations are emphasized: 1) A low sample, due to rarity of diagnosis in absolute numbers and to the low number of survivors - resultant of complications arising from the late diagnosis and to low socioeconomic conditions of children; 2) WISC-III low sensitivity to detect subtle neuropsychological deficits, since this instrument was not designed for children in pathological conditions. This aspect leads to the need to complement the assessment of this clinical population with neuropsychological tests that cover specific cognitive dimensions, as well as with tasks adapted to the study of children with severe brain injuries. In addition, data from neuroimaging tests, in particular, fractional anisotropy, should be obtained to investigate the presence of white matter injury among children exposed to cranial radiation therapy.

Future investigations should overcome the limitations mentioned above, so as to enable relevant contributions to future proposition of differentiated services to children with cancer - seeking to contribute to their quality of life and ensuring them the necessary potential for their development.

\section{Acknowledgements}

We thank Joao Carlos Alencar for the valuable assistance in data analyses.

This study was supported by two Brazilian research funding agencies: Coordenação de Aperfeiçoamento de Pessoal de Nível Superior and Conselho Nacional de Desenvolvimento Científico e Tecnológico. 


\section{References}

1. INCA (2014) Estimativa 2014: Incidência de Câncer no Brasil. Rio de Janeiro: Ministério da Saúde/ Instituto Nacional do Câncer.

2. Hazin I, Dellatolas G, Garcia D, Pedrosa F, Pedrosa A (2011) Intellectual impairment after treatment for medulloblastoma and astrocytoma in childhood: the Brazilian experience. J Pediatr Hematol Oncol 33: 506-515.

3. Lins M (2005) Fatores associados ao retardo no diagnóstico das leucemias agudas na infância em um serviço de referência no nordeste do Brasil. Dissertação de mestrado, Instituto Materno-Infantil Professor Fernando Figueira, Pernambuco, Recife, Brasil.

4. Moxon-Emre I, Bouffet E, Taylor MD, Laperriere N, Scantlebury N, et al. (2014) Impact of Craniospinal Dose, Boost Volume, and Neurologic Complications on Intellectual Outcome in Patients With Medulloblastoma. J Clin Oncol .

5. Nathan PC, Patel SK, Dilley K, Goldsby R, Harvey J, et al. (2007) Guidelines for identification of, advocacy for, and intervention in neurocognitive problems in survivors of childhood cancer: a report from the Children's Oncology Group. Arch Pediatr Adolesc Med 161: 798-806.

6. Butler RW, Haser JK (2006) Neurocognitive effects of treatment for childhood cancer. Ment Retard Dev Disabil Res Rev 12: 184-191.

7. Mabbott DJ, Penkman L, Witol A, Strother D, Bouffet E (2008) Core neurocognitive functions in children treated for posterior fossa tumors. Neuropsychology 22: 159-168.

8. Vaquero E, Gómez CM, Quintero EA, González-Rosa JJ, Márquez J (2008) Differential prefrontal-like deficit in children after cerebellar astrocytoma and medulloblastoma tumor. Behav Brain Funct 4: 18.

9. Aarsen FK, Paquier PF, Arts WF, Van Veelen ML, Michiels E, et al. (2009) Cognitive deficits and predictors 3 years after diagnosis of a pilocytic astrocytoma in childhood. J Clin Oncol 27: 3526-3532.

10. Rondinelli PIP (2003) Neoplasias astrocíticas de baixo grau e neoplasias neuronais mistas e neuroniogliais. In Furrer AA, Osorio CAM, Rondinelli PIP, Junior PIS (orgs.), Neurologia oncológica pediátrica: manejo multidisciplinar das neoplasias do sistema nervoso central da infância (pp. 245-252). São Paulo: Lemar.

11. Gottardo NG, Gajjar A (2006) Current therapy for medulloblastoma. Curr Treat Options Neurol 8: 319-334.

12. Pui CH, Carroll WL, Meshinchi S, Arceci RJ (2011) Biology, risk stratification, and therapy of pediatric acute leukemias: an update. J Clin Oncol 29: 551-565.

13. Massimino M, Cefalo G, Riva D, Biassoni V, Spreafico et al. (2012) Longterm results of combined preradiation chemotherapy and age-tailored radiotherapy doses for childhood medulloblastoma. Journal of Neuro Oncology 108: 163-171.

14. Anderson FS, Kunin-Batson AS (2009) Neurocognitive late effects of chemotherapy in children: the past 10 years of research on brain structure and function. Pediatr Blood Cancer 52: 159-164.

15. Temming P, Jenney ME (2010) The neurodevelopmental sequelae of childhood leukaemia and its treatment. Arch Dis Child 95: 936-940.

16. Baron Nelson M, Compton P, Patel SK, Jacob E, Harper R (2013) Central nervous system injury and neurobiobehavioral function in children with brain tumors: a review of the literature. Cancer Nurs 36: E31-47.

17. Gomes ER, Leite DS, Garcia D, Maranhão S, Hazin I (2012) Neuropsychological profile of patients with acute lymphoblastic leucemia. Psychology \& Neuroscience 5 : 175-182.

18. Reddick WE, Taghipour DJ, Glass JO, Ashford J, Xiong X, et al. (2014) Prognostic factors that increase the risk for reduced white matter volumes and deficits in attention and learning for survivors of childhood cancers. Pediatr Blood Cancer 61: 1074-1079.

19. Rodgers SP, Trevino M, Zawaski JA, Gaber MW, Leasure JL (2013) Neurogenesis, exercise, and cognitive late effects of pediatric radiotherapy. Neural Plast 2013: 698528.

20. Palmer SL, Glass JO, Li Y, Ogg R, Qaddoumi I, et al. (2012) White matter integrity is associated with cognitive processing in patients treated for a posterior fossa brain tumor. Neuro Oncol 14: 1185-1193.
21. Watanabe S, Azami Y, Ozawa M, Kamiya T, Hasegawa D, et al. (2011) Intellectual development after treatment in children with acute leukemia and brain tumor. Pediatr Int 53: 694-700.

22. Brinkman TM, Reddick WE, Luxton J, Glass JO, Sabin ND, et al. (2012) Cerebral white matter integrity and executive function in adult survivors of childhood medulloblastoma. Neuro Oncol 14 Suppl 4: iv25-36.

23. Rieken S, Mohr A, Habermehl D, Welzel T, Lindel K, et al. (2011) Outcome and prognostic factors of radiation therapy for medulloblastoma. Int J Radiat Oncol Biol Phys 81: e7-7e13.

24. Duffner PK (2010) Risk factors for cognitive decline in children treated for brain tumors. Eur J Paediatr Neurol 14: 106-115.

25. Palmer SL, Armstrong C, Onar-Thomas A, Wu S, Wallace D, et al. (2013) Processing speed, attention, and working memory after treatment for medulloblastoma: an international, prospective, and longitudinal study. J Clin Oncol 31: 3494-3500.

26. Bonner MJ, Hardy KK, Willard VW, Gururangan S (2009) Additional evidence of a nonverbal learning disability in survivors of pediatric brain tumors. Children's Health Care $38: 49-63$.

27. Palmer SL, Reddick WE, Gajjar A (2007) Understanding the cognitive impact on children who are treated for medulloblastoma. J Pediatr Psychol 32: 1040-1049.

28. Hazin I, Leitão S, Garcia D, Gomes E, Lemos C (2010) Contribuições da Neuropsicologia de Alexsandr Romanovich Luria para o debate contemporâneo sobre relações mente-cérebro. Mnemosine 6:88-110.

29. Mello CB, Miranda MC, Feldman C, Sinnes EG, Barbosa T, Beltrami MC et. al. (2006) Abordagem interdisciplinar em lesões encefálicas na infância. In C. B. Mello, M. C. Miranda \& M. Muszkat (orgs.), Neuropsicologia do desenvolvimento: conceitos e abordagens 223-236) São Paulo: Memnon.

30. Cantelmi D, Schweizer TA, Cusimano MD (2008) Role of the cerebellum in the neurocognitive sequelae of treatment of tumours of the posterior fossa: an update. Lancet Oncol 9: 569-576.

31. Buizer AI, de Sonneville LM, Veerman AJ (2009) Effects of chemotherapy on neurocognitive function in children with acute lymphoblastic leukemia: a critical review of the literature. Pediatr Blood Cancer 52: 447-454.

32. Rønning C, Sundet K, Due-Tønnessen B, Lundar T, Helseth E (2005) Persistent cognitive dysfunction secondary to cerebellar injury in patients treated for posterior fossa tumors in childhood. Pediatr Neurosurg 41: 15-21.

33. Cunha JA (2000) Escalas Wechsler In Cunha JA (org.), Psicodiagnóstico V (pp. 529-602). Porto Alegre: Artmed.

34. Walsh KS, Paltin I, Gioia GA, Isquith P, Kadan-Lottick NS, et al. (2014) Everyday executive function in standard-risk acute lymphoblastic leukemia survivors. Child Neuropsychol .

35. Krull K, Zhang N, Santucci A, Srivastava D, Krasin M, et al. (2013) Longterm decline in intelligence among adult survivors of childhood acute lymphoblastic leukemia treated with cranial radiation. Blood Journal $122: 550-553$

36. Peterson CC, Johnson CE, Ramirez LY, Huestis S, Pai AL, et al. (2008) A meta-analysis of the neuropsychological sequelae of chemotherapy-only treatment for pediatric acute lymphoblastic leukemia. Pediatr Blood Cancer 51: 99-104.

37. Stargatt R, Rosenfeld JV, Anderson V, Hassall T, Maixner W, et al. (2006) Intelligence and adaptive function in children diagnosed with brain tumour during infancy. J Neurooncol 80: 295-303.

38. Moura R , Haase V (2010) Transtorno Não-Verbal de Aprendizagem: aspectos neuropsicológicos. In: do Valle LER, Assumpção Jr. F, Wajnsztejn R, Malloy-Diniz LF, Aprendizagem na atualidade: neuropsicologia e desenvolvimento na inclusão (pp. 135-146). Ribeirão Preto: Novo Conceito Editora.

39. Fry AF, Hale S (2000) Relationships among processing speed, working memory, and fluid intelligence in children. Biol Psychol 54: 1-34.

40. Figueiredo V (2000) WISC III. In Cunha JA (Org.), Psicodiagnóstico V. Porto Alegre: Artmed 603-614. 
Citation: Hazin I, Garcia D, Gomes E, Garcia BB, Sunaly D, et al. (2014) Treatment Related Cognitive Impairment in Pediatric Oncology Patients: A Brazilian Experience. J Nucl Med Radiat Ther 5: 174. doi:10.4172/2155-9619.1000174

Page 9 of 9

41. Moleski M (2000) Neuropsychological, Neuroanatomical, and Neurophysiological Consequences of CNS Chemotherapy for Acute Lymphoblastic Leukemia. Archives of Clinical Neuropsychology 15: 603-630.

42. Palmer SL, Leigh L (2009) Survivors of pediatric posterior fossa tumors: cognitive outcome, intervention, and risk-based care. Eur J Oncol Nurs 13: 171-178.

43. Cazé MO, Bueno D, Santos MEF (2010) Estudo referencial de um protocolo quimioterápico para leucemia linfocítica aguda infantil. Revista do Hospital de Clínicas de Porto Alegre 30: 5-12.

44. Holland A (2013) Neuropsychological comparison of pediatric medulloblastoma and pilocytic astrocytoma: existing knowledge and future directions. The new school psychology bulletin $10: 1-20$
45. Krawczuk-Rybak M, Grabowska A, Protas PT, Muszynska-Roslan K, Holownia A, et al. (2012) Intellectual functioning of childhood leukemia survivors - relation to Tau protein - a marker of white matter injury. Advances in Medical Sciences 57: 266-272.

46. Blomstrand M, Broading N, Rosenschöld P, Vogellus I, Merino G, et.al. (2012) Estimated clinical benefit of protecting neurogenesis in the developing brain during radiation therapy for pediatric medullobalstoma. Neuro-oncology 14 : 882-889. 